\title{
Strong limit on the spatial and temporal variations of the fine-structure constant
}

\author{
T. D. Le \\ Department of Physics, Center for Natural Science, Vietnam \\ email: ldthong@hotmail.com
}

\begin{abstract}
Observed spectra of quasars provide a powerful tool to test the possible spatial and temporal variations of the fine-structure constant $\alpha=e^{2} / \hbar c$ over the history of the Universe. It is demonstrated that high sensitivity to the variation of $\alpha$ can be obtained from a comparison of the spectra of quasars and laboratories. We reported a new constraint on the variation of the finestructure constant based on the analysis of the optical spectra of the fine-structure transitions in $[\mathrm{NeIII}],[\mathrm{NeV}],[\mathrm{OIII}],[\mathrm{OI}]$ and $[\mathrm{SII}]$ multiplets from 14 Seyfert 1.5 galaxies. The weighted mean value of the $\alpha$-variation derived from our analysis over the redshift range $0.035<z<0.281$ $\Delta \alpha / \alpha=(4.50 \pm 5.53) \times 10^{-5}$. This result presents strong limit improvements on the constraint on $\Delta \alpha / \alpha$ compared to the published in the literature
\end{abstract}

Keywords. galaxies: fundamental parameters (classification, colors, luminosities, masses, radii, etc.); cosmology: observations; methods: data analysis

\section{Introduction}

The interesting problem of time variation of the fundamental constants such as the finestructure constant,

$$
\alpha=\frac{e^{2}}{4 \pi \epsilon_{0} \hbar c}
$$

in the expanding Universe was questioned by Dirac and Milne (Dirac 1973; Milne 1937). This subject is current interest because of the new possibilities opened up by the contemporary theories, which lead us to unify the fundamental interactions of nature. Astrophysical estimates of the $\alpha$ is based on the comparison of the line centers in the absorption/emission spectra of astronomical objects and the corresponding laboratory values (Bahcall et al. 2004; Dzuba et al. 1999; Le 2014). The AD method is based on the fine-structure splitting. The splitting ratios $s(t)$,

$$
s(t)=\frac{\lambda_{2}(t)-\lambda_{1}(t)}{\lambda_{2}(0)+\lambda_{1}(0)}
$$

at two different epochs, gives the different values in $\alpha$ between them two epochs. It is shown that,

$$
\frac{\Delta \alpha}{\alpha}(t)=\frac{1}{2}\left[\frac{\left(\lambda_{2}(t)-\lambda_{1}(t)\right) /\left(\lambda_{2}(t)+\lambda_{1}(t)\right)}{\left(\lambda_{2}(0)-\lambda_{1}(0)\right) /\left(\lambda_{2}(0)+\lambda_{1}(0)\right)}-1\right],
$$

where $\lambda_{2}(t)$ and $\lambda_{1}(t)$ are the wavelengths obtained from the quasar observations, and $\lambda_{2}(0)$ and $\lambda_{1}(0)$ the laboratory wavelengths. The best recent result using AD method applied to quasar absorption lines is $\Delta \alpha / \alpha=(-0.5 \pm 1.3) \times 10^{-5}$ at $0.2<z<4.2$ (Bahcall et al. 2004). 
Another method is the MM method, which was developed by Dzuba et al.(1999). This is dependent on the rest wavelengths to the variation of $\alpha$ parameter by using the fitting function,

$$
\omega=\omega_{0}+q_{1} x+q_{2} y
$$

where $\omega_{0}$ and $\omega$ are the vacuum wave number measured in the laboratory and in the absorption system at redshift $\mathrm{z}$, and the parameters $x$ and $y$ are defined as

$$
\begin{aligned}
& x=\left(\frac{\alpha_{z}}{\alpha_{0}}\right)^{2}-1 \\
& y=\left(\frac{\alpha_{z}}{\alpha_{0}}\right)^{4}-1 .
\end{aligned}
$$

The best recent result using MM method applied to KECK/HIRES data were $\Delta \alpha / \alpha=$ $(-0.57 \pm 0.10) \times 10^{-5}$ at $0.2<z<3.7$. This method simultaneously analyzes many doublets of many atomic species and due to the large number of linesused, gives an order magnitude improvement in the measurement of $\Delta \alpha / \alpha$ compared with the AD. However, several uncertainties and possible systematics would be some unnoticed systematic effects and might challenge that interpretation and it is very difficult to determine the systematic effects and the true values of $\Delta \alpha / \alpha$.

A new method is needed to improve the disadvantages of these methods to investigate spatial and temporal variation in $\alpha$ using the absorption/ emission lines (Bahcall et al. 2004; Dzuba et al. 1999).

\section{Astrophysical data tests of $\Delta \alpha / \alpha$}

In spectra of QSOs systems, they include almost resonance lines of the ions, and others corresponding to the transitions ground state. The separation between energy levels caused by fine- splitting is proportional to $\alpha^{4}$ with leading to term of energy level being proportional to $\alpha^{2}$. The spectroscopic observations of gas clouds present ideal laboratories in which to search for any spatial and temporal of $\alpha$, which is based on the fact that the energy of each line transition depends individually on a change in $\alpha$.

Therefore, we applied the new method that was developed by Le (2014) to improve the disadvantages of the $\mathrm{AD}$ and MM methods to search for spatial and temporal variation of the fine structure constant. This method allows us to estimate $\alpha$ values at early stages of the Universe evolution by using the fine-splitting doublets and multiplets seen in quasar spectra and has the advantage that it is more transparent and less subject to systematics is approximately written as

$$
\frac{\Delta \alpha}{\alpha}=\frac{1}{2}\left(\frac{\frac{1}{2}\left(\frac{\lambda_{2}(t)}{\lambda_{1}(t)}\right)-1}{\frac{1}{2}\left(\frac{\lambda_{2}(0)}{\lambda_{1}(0)}\right)-1}-1\right)
$$

provided that $\Delta \alpha / \alpha$ is very small.

Thus, comparing the relative of measured wavelengths $\lambda_{1}(t)$ and $\lambda_{2}(t)$ in an absorption (or emission) system at the redshift $\mathrm{z}$ with the corresponding laboratory values, one can directly infer the possible variation of $\alpha$ at different epochs, space-time points, regions of the Universe and the present value.The observed wavelengths from quasars will be determined through the cosmological redshift parameteras $1+z=\lambda_{\text {observ }} / \lambda_{\text {emiss }}$, where $\lambda_{\text {emiss }}$ is the wavelength at the moment of emission (absorption) by a distant source in 
the past and $\lambda_{\text {observ }}$ is the observed value. Thus, this method can be used to study not only possible effect of the time variability of the fine-structure constant at present but also at earlier epochs of the Universe, when they were causally disconnecte.

In this study, we have applied a new method to analysis the optical spectra of the finestructure transitions in [NeIII], [NeV], [OIII], [OI] and [SII] multiplets from 14 Seyfert 1.5 galaxies to constrain past variations in $\alpha$ (Grupe et al. 2005). This approach eliminates the largest systematic errors present in other determinations of $\alpha$ and provides estimates of the remaining statistical and systematic errors. This method includes $\alpha$-independent line ratios which can identify the true size of statistical and systematic errors.

\section{Conclusion}

Our result is $\Delta \alpha / \alpha=(4.50 \pm 5.53) \times 10^{-5}$ over the redshift range $0.035<z<0.281$. Our result corresponds to two orders of magnitude more sensitive than that using the same data derived by Grupe et al. (2005). This result is nearly two order magnitude improvements on the constraint on / compared with the AD method Bahcall et al. (2004) and an order compared with MM method (Dzuba et al. 1999). Our results consist with the results coming from Oklo natural nuclear reactor (Shylakher 1976). The spatial gradient in values of $\alpha$ are in progress.

\section{References}

Bahcall J. N. et al. 2004, ApJ 600, 520

Dirac A. M. 1973, Nature 139, 323

Dzuba V. A. et al. 1999, PRL 82, 888

Milne E. A. 1937, Proc. R. Soc. Ser. A. 158, 324

Le T. D. 2014, AIP Conf. Proc. 1594, 23

Grupe D. et al., 2005, AJ 130, 255

Shylakher A. I., 1976, Nature 264, 340 